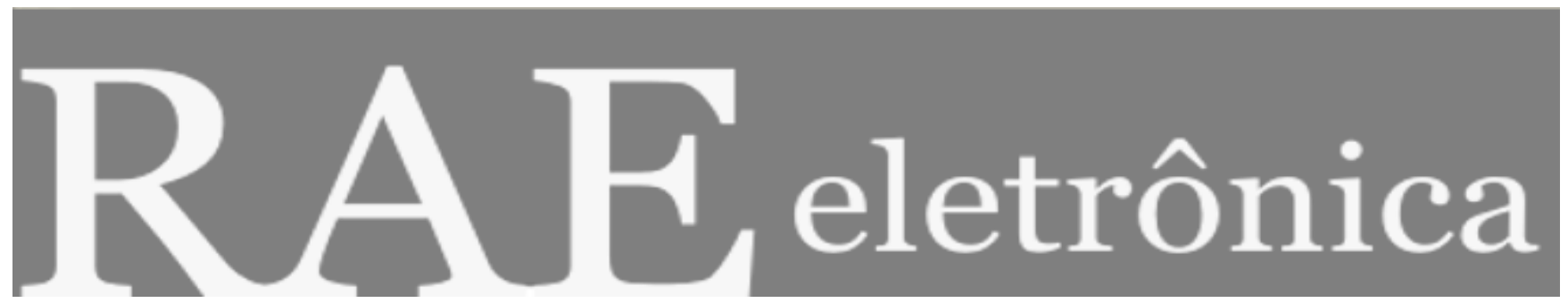

\title{
RACIONALIDADE E INCORPORAÇÃO DE TECNOLOGIA EM SAÚDE: A EXPERIÊNCIA DE UM HOSPITAL DE ALTA COMPLEXIDADE EM S. PAULO
}

Por:

Ana Carolina S. Queiroz

Antonio Pires Barbosa

RAE-eletrônica, Volume 2, Número 1, jan-jun/2003.

http://www.rae.com.br/eletronica/index.cfm?FuseAction=Artigo\&ID=1895\&Secao $=2^{\circ} \mathrm{PWC} \&$ Volume $=2 \&$ Numero $=$ $1 \& A n o=2003$

CCopyright, 2002, RAE-eletrônica. Todos os direitos, inclusive de tradução, são reservados. É permitido citar parte de artigos sem autorização prévia desde que seja identificada a fonte. A reprodução total de artigos é proibida. Os artigos só devem ser usados para uso pessoal e nãocomercial. Em caso de dúvidas, consulte a redação: redacao@rae.com.br.

A RAE-eletrônica é a revista on-line da FGV-EAESP, totalmente aberta e criada com o objetivo de agilizar a veiculação de trabalhos inéditos. Lançada em janeiro de 2002, com perfil acadêmico, é dedicada a professores, pesquisadores e estudantes. Para mais informações consulte o site www.rae.com.br/eletronica.

RAE-eletrônica ISSN 1676-5648

(C)2002 Editora: Fundação Getulio Vargas - Escola de Administração de Empresas de São Paulo.

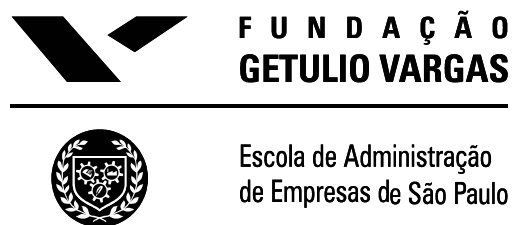




\title{
RACIONALIDADE E INCORPORAÇÃO DE TECNOLOGIA EM SAÚDE: A EXPERIÊNCIA DE UM HOSPITAL DE ALTA COMPLEXIDADE EM S.PAULO.
}

\author{
Ana Carolina S. Queiroz \\ Doutora em Administração de Empresas pela FGV-EAESP. \\ E-mail: carol@innovaction.com.br \\ Endereço: Rua Dr. Sodré, 122 Conj. 125/126 Vila Olímpia - São Paulo, SP, 04535-110 \\ Interesses de pesquisa: Teoria das organizações, Recursos Humanos, Tecnologia e Estratégia.
}

\section{Antonio Pires Barbosa}

Mestre em Administração de Empresas e Doutorando na FGV-EAESP.

E-mail: rbe.pires@terra.es

Endereço: Av. Nove de Julho, 2029 - Bela Vista - São Paulo, SP, 01313-902

Interesses de pesquisa: Tecnologia, Tecnologia Médica, Teoria das Organizações e Planejamento.

\section{RESUMO}

O artigo analisa a evolução do modelo decisório utilizado por uma instituição hospitalar de grande porte em S.Paulo com o intuíto de demonstrar a necessidade de desenvolvimento de uma matriz de informações que subsidiem o processo. Questões relacionadas ao mercado comprador de seus serviços, às características epidemiológicas da população, às indicações de uso da tecnologia pelo corpo médico bem como à capacidade interna de absorção pela organização devem ser tomadas em consideração além do estudo de retornos financeiros. Propõe, ainda, o estabelecimento de um modelo de classificação dos investimentos destinados à tecnologia médica em organizações de saúde, que pressupõe a consideração de fatores como a condição de segurança associada à tecnologia, sua essencialidade e rentabilidade para o hospital.

\section{PALAVRAS-CHAVE}

Planejamento. Tecnologia médica. Processo decisório. Informações. Critérios.

\begin{abstract}
The arcticle analyses the evolution of the decision model used at a large hospital institution in Sao Paulo aiming to demonstrate the need for developing a information network which subsides the process. Issues regarding the market of costumers of its services, the epidemiologic characteristics of the population, the indications of thechnology use by the medical staff as well as the internal absorption capacity of the institution must be considered besides the study of the financial return. It also proposes the settlement of a model to classify the investment applied to medical technology at healthcare organizations that assumes a consideration of factors like safety conditions associated to the technology, its priority and rentability to the hospital.
\end{abstract}

\section{KEYWORDS}

Planning. Medical Technology. Decision Proces. Information. Criterion. 


\section{INTRODUÇÃO}

Nos últimos anos tem-se assistido a uma transformação profunda nos sistemas de saúde no mundo todo. Novos conhecimentos médicos, diferentes abordagens terapêuticas, a conscientização por parte das populações acerca dos fatores de risco de agravo à saúde e a crescente agregação de ferramentas de informação à prática assistencial tem possibilitado a extensão do atendimento a situações que até algum tempo atrás insolúveis. O investimento no desenvolvimento de novas tecnologias e fármacos, cujas capacidades de resolução diagnóstica e terapêutica se revelam de maior impacto, por setores industriais envolvidos com a saúde, tem se acentuado, especialmente nas sociedades desenvolvidas onde a pressão dos usuários sobre o sistema traz a necessidade de novas formas de atuação e o reexame da matriz de produção de serviços médicos-assistenciais (Andrews, 1994).

A concentração da produção de serviços sobre o hospital passa a ser questionada dentro de uma perspectiva de transformação social que tende a considerar, em um contexto estratégico, os principais fatores relacionados à manutenção da sobrevivência e das condições de produção e consumo. Fatores como o envelhecimento da população, a queda nas taxas de natalidade e fertilidade, a urbanização, o aumento da violência, a complexidade do perfil nosológico das populações, o aumento da freqüência de doenças crônico-degenerativas, o aumento do desemprego e as distorções das distribuições de renda tornam-se, em conseqüência, parte das preocupações com que os responsáveis pelo planejamento têm que se defrontar na construção de um modelo assistencial caracterizado por um alto grau de complexidade, qualidade e custos que garantam o acesso do maior contingente de pessoas (Porter O’Grady, Wilson 1995).

Neste cenário, emergem tendências de reorganização da assistência que privilegiam novas premissas e conceitos e impactam diretamente as formas tradicionalmente aceitas como as mais adequadas para uma boa prática. É relevante, por exemplo, citar os movimentos pela adequação dos custos, pela valorização da evidência no exercício médico, pelo desenvolvimento de novas formas de atendimento extra-hospitalares, pela redução do número de leitos hospitalares, pela busca de tecnologias de alta capacidade resolutiva ambulatorial e pela participação da sociedade na discussão de formas mais econômicas e efetivas de atuação (De Wolf e Giloth, 1998; Mycek, 1998).

Da mesma forma, a discussão que se estabelece acerca atuação e dos papéis reservados aos principais atores envolvidos traz em sua essência questões que envolvem o reposicionamento da sociedade em relação aos aspectos funcionais do sistema e aos seus mecanismos reguladores da qualidade e da segurança (Banga, 1997). Neste sentido, é possível a identificação de , pelo menos , sete novas tendências decorrentes desta nova concepção:

- O controle das formas de financiamento da atividade, quer em países com predomínio de modelos públicos, quer de modelos privados, tendo em vista o atendimento de princípios gerais de utilidade e adequação dos serviços.

- O controle da qualidade dos serviços prestados, envolvendo as visões dos prestadores, dos usuários e dos administradores.

- O monitoramento do parque de tecnologia e do seu grau de resolutividade. 
- A revisão das formas e conteúdos dos processos de educação e formação dos profissionais de saúde.

- A inserção de novas formas de abordagem terapêutica, dentro de um princípio de continuidade do cuidado, com estratificação das atividades de prevenção, atuação sobre a doença, reabilitação e re-inserção social.

- O estabelecimento de um novo papel educativo e formador de opinião por parte da mídia, que passa a se envolver nas questões relacionadas ao setor, com ênfase sobre a tecnologias e os eventos de não conformidade.

- O redesenho do papel do poder público, que passa a ter uma função eminentemente reguladora e disciplinadora das relações entre os agentes.

Em um contexto de tal ordem, a questão da decisão de aquisição de novas tecnologias médicas assume importância crucial por parte dos gestores de organizações de saúde, posto que deve se considerar informações que possibilitem uma melhor utilização dos recursos disponíveis. Atributos relacionados aos vários agentes envolvidos, à capacidade resolutiva, ao potencial de redução de custos e à cultura e formação dos envolvidos com a produção passam a fazer parte do processo decisório e não apenas os aspectos financeiros vinculados à opção de compra ou financiamento de determinada incorporação. Em conseqüência, selecionar opções que propiciem as melhores relações custo benefício, os melhores desempenhos operacionais e que abram novas possibilidades de desenvolvimento de conhecimentos pode vir a demandar novos modelos de avaliação em que estes fatores estejam contemplados.

Neste artigo, procura-se analisar o exemplo de um hospital de grande porte em S.Paulo, reconhecido pela complexidade de seu parque tecnológico, com o intuito de mostrar a evolução do seu modelo decisório de incorporação e a relevância que as informações relativas a mercado, capacidade resolutiva, características intrínsecas da tecnologia e potencial de retorno financeiro passaram a ter na seleção de opções. Da mesma forma, discutir os papéis dos médicos envolvidos com a utilização e o manejo da tecnologia como elementos fundamentais na decisão de agregação de novos procedimentos tecnológicos pela organização de saúde.

$\mathrm{O}$ artigo apresenta uma estrutura onde se discute no próximo tópico as características gerais dos elementos participantes do sistema de saúde; no tópico três as observações acerca do modelo utilizado pelo hospital e sua evolução, no tópico quatro a descrição simplificada de uma classificação que inclue os principais critérios a serem considerados e por fim a conclusão.

\section{OS ATORES ENVOLVIDOS E SUAS CARACTERÍSTICAS}

\section{O Paciente/ Cliente do Sistema.}

Saúde é um bem intangível. Somente percebe-se sua importância e valor quando o equilíbrio na dinâmica saúde-doença é quebrado. Vincula-se diretamente à capacidade que os indivíduos têm de manter sua sobrevivência e a continuidade da espécie (Chaves, 1982). Os avanços da ciência médica e a melhoria das condições de vida das populações implicaram em mudanças radicais nas suas 
composições, com consequências para a sociedade e sua produção (Andrews, 1994).

Estas passam, com os modelos de desenvolvimento da revolução tecnológica, a apresentarem profundas mudanças em seus comportamentos. $\mathrm{O}$ aumento da concentração urbana, as mudanças de hábitos de consumo e as mudanças na distribuição de renda levam os indivíduos a uma maior preocupação com aspectos físicos de seu bem-estar. Da mesma forma, as possibilidades de maiores acessos a informações sobre saúde, doença e seus desequilíbrios tornam os indivíduos mais conscientes e isto, aliado a uma revisão dos conceitos do direito do consumidor, traz uma nova postura individual face à forma de atuação do sistema de saúde (Baig, 1994).

Tradicionalmente, o médico era visto como uma entidade semidivina e inacessível. Esta característica felizmente tem apresentado mudanças sensíveis. O paciente adquire a capacidade de questionar e criticar as atitudes e práticas profissionais desta área. A sociedade, cria em consequência, mecanismos de gerenciar estas relações com uma ênfase acentuada na mediação e aferição da qualidade e da ética envolvida (Barbosa, 1995, Picchiai, 1998).O paciente/cliente deixa de assumir uma posição passiva e passa a interferir diretamente com os processos aos quais é submetido e observa-se uma mudança nas obrigações estabelecidas aos profissionais de saúde que antes eram de meio e passam a ser de fins, dos resultados para a vida. Esta tendência se acentua a ponto de modelos sofisticados de gerenciamento de riscos por má-prática serem incorporados à atividade.

\section{O Prestador de Serviços}

O hospital constitui o elemento central da assistência à saúde pela concentração de recursos e competências específicas de atendimento, sendo o médico o principal tutor da assistência. A relação que se estabelece com este tipo de organização é de dependência, e este fenômeno está nas raízes do sentimento opressivo associado ao ambiente hospitalar. Entretanto, com o desenvolvimento de novas abordagens muitas práticas coercitivas da instituição hospitalar passam a receber críticas a partir de seus próprios profissionais. Isto acaba por levar ao desenvolvimento de um novo modelo de atendimento em que alguns princípios assumem maior importância (Anderson, 1992), tais como:

- O privilegio da atenção ambulatorial e domiciliar.

- A redução dos tempos de internação e o gerenciamento dos fatores de riscos a ela associados.

- A inserção na pesquisa médica e de tecnologia de parâmetros que enfoquem o atendimento fora do ambiente hospitalar.

- A instituição de novo desenho legal que promova o respeito a padrões de comportamentos éticos, especialmente em relação ao respeito à vontade do paciente e ao seu direito de informação.

Estas asserções estão promovendo uma revolução dos costumes dos profissionais de saúde, especialmente na medicina. A profissão médica tem se constituído por razões técnicas e históricas na profissão de saúde mais valorizada. Todas as medidas e comportamentos observados nos profissionais de saúde são regularmente orientados pelo profissional médico. No ambiente do hospital, reserva-se ao médico o papel de herói institucional. $\mathrm{Na}$ realidade, devido ao alto grau de exigência presente na assistência tais posturas são naturais. Além disso, a condição de resolutividade e o atendimento de 
casos de maior dificuldade e complexidade clínica, acaba fomentando a imagem heróica do profissional médico (Landmann, 1982).

Entretanto é importante estabelecer que as mudanças em curso também têm possibilitado o aparecimento de um novo traço importante: $O$ de atuação multidisciplinar e multiprofissional. Isto ocorre em grande medida pela impossibilidade do médico assumir toda a operação e controle da assistência que as novas tecnologias e processos tem trazido ao setor (Tonges,1992).

\section{A Fonte Financiadora}

No Brasil, é possível identificar três formas de financiamento do sistema de saúde. O Sistema Único de Saúde - SUS, financiado pelo Estado, o Sistema Supletivo de Assistência, financiado pelo recolhimento de contribuições contratadas e o autofinanciamento (Constituição Federal, 1988). A presença concomitante destes modelos em um mesmo mercado de assistência, contudo, é responsável por uma série de desvios de função. No fundo, pode-se inferir que o Estado tem uma capacidade limitada de inversão de recursos no sistema em razão das suas opções econômicas. O que se pode observar é uma tendência de privatização das ações de saúde via alavancagem do sistema supletivo que está mais diretamente vinculado ao ambiente da produção (cerca de $70 \%$ dos planos de saúde relaciona-se a empresas e trabalhadores da economia formal)

As organizações financiadoras, em conseqüência das mudanças ocorridas, passam a incorporar (o Estado inclusive) novas formas de financiamento tentando obter uma redução dos custos com os serviços prestados. A utilização de ferramentas estatísticas, de economia da saúde e de medicina baseada em evidências desemboca em novas práticas como, por exemplo, o Managed Care, o Capitation, a implantação de gate-keepers cuja principal função é a redução de custos. Os aspectos da qualidade assistêncial e da obtenção de resultados beneficos ao paciente passam a ter importância secundária neste modelo (Soper et all, 1995).

\section{A Indústria Farmacêutica}

Um dos setores onde ocorrem os maiores investimentos em pesquisa e desenvolvimento no mundo é o químico-farmacêutico. Simultaneamente, a dominação do setor é exercida por poucos grupos multinacionais, predominantemente de origem européia e americana. $\mathrm{O}$ volume de lançamentos de novos produtos é regulado nestes países por agências controladoras da eficácia e efetividade que seus produtos apresentam. As sociedades onde se localizam as matrizes destes grupos conseguiram chegar a um estado de regulação da atividade bastante rigoroso na validação e aceitação dos fármacos. Outro dado importante é que, nestes países, as leis de patente e propriedade industrial acabam por limitar o tempo de exclusividade a 15 anos de registro. Com um tempo médio de liberação dos medicamentos longo, na prática estas indústrias usufruem preços elevados por um período relativamente curto, após o qual passam a sofrer concorrência de laboratórios especializados na produção de genéricos de equivalente eficácia .

\section{A Indústria Eletromédica}

À semelhança do que ocorre na indústria farmacêutica, a área de tecnologia médica também apresenta uma forte concentração de empresas multinacionais. A diferença é que nesta área, as empresas mais importantes são da área eletro - eletrônicas e saúde não constitui seu principal negócio. 
O investimento em pesquisa e desenvolvimento, no entanto, é também elevado e um dos objetivos estratégicos destas empresas ao investirem nestas áreas é a melhoria de sua imagem. Logo, as margens de comercialização dos seus produtos médicos não são em essência diferentes dos outros produtos que compõem seu port-fólio.

Entretanto, a incorporação de tecnologia, quer diagnóstica, quer terapêutica foi uma das facetas da assistência que passou pelas maiores transformações nos últimos anos. A obsolescência dos equipamentos reduziu seus ciclos para períodos muito curtos (Augier, 2000,Bronzino, 1995). A veiculação pela mídia das novidades em tecnologias imprime uma demanda por novos métodos por parte dos consumidores que acaba por encarecer a assistência. Por outro lado, a tecnologia também tem propiciado, especialmente nos últimos anos, uma mudança acentuada no modelo de atendimento com redução da permanência em hospitais, com o crescimento do atendimento ambulatorial mai complexo e coma introdução de novas formas de atendimento .

\section{O Poder Público}

Como agente regulador, o poder público tem se pautado, desde a última década pela criação de legislação que modeladora e balizadora do comportamento dos demais agentes. Por exemplo, isto pode ser notado com a reforma legal do sistema supletivo de saúde e com a criação da AnVisa-Agência Nacional de Vigilância Sanitária com poder fiscalizatório sobre a produção de produtos usados em saúde. Em princípio, o elemento norteador destas mudanças tem sido o de disponibilizar à sociedade estruturas e processos que lhe permitam controlar a atividade dos diversos agentes.

Mais recentemente, com o conhecimento mais detalhado acerca do risco associado a determinados comportamentos, este agente tem também se empenhado na educação da população quanto ao gerenciamento destes fatores de risco. Esta tendência tem forte vinculação com as mudanças ocorridas no modelo de formação do profissional do setor que passa a ter maior consciência de seus papéis e responsabilidades, influenciando a sociedade e o poder público no sentido das mudanças necessárias.

\section{O MODELO DESENVOLVIDO POR UM HOSPITAL DE GRANDE PORTE EM S.PAULO QUE DISPÕE DE UM COMPLEXO PARQUE TECNOLÓGICO}

Até meados dos anos 70 a prática médica, do ponto de vista de utilização de tecnologia, baseava-se em um modelo de baixa sofisticação. A propedêutica tecnológica se restringia a técnicas que àquela época já apresentavam restrições resolutivas. Entretanto, com o desenvolvimento de novas aplicações nas indústrias do setor novas metodologias emergiram com a ampliação da capacidade. Por exemplo, é desta época o desenvolvimento da tomografia computadorizada e a utilização de fibras óticas na endoscopia. Os hospitais estruturavam processos assistenciais de baixa complexidade desenvolveram o interesse nas novas tecnologias e modificaram sua produção no sentido de atender seus mercados e médicos com novos equipamentos, técnicas e abordagens terapêuticas.

Uma das conseqüências foi o encarecimento da assistência e a competição pela posição de vanguarda por parte de alguns hospitais que passaram a contar com uma visão mercadológica de seus negócios. Por algum tempo o conceito de assistência de qualidade desloca-se da competência médica para a sofisticação dos equipamentos e do potencial de inserção em mídia que suas aquisições propiciavam. 
Entretanto, o processo decisório de incorporação tecnológica nesta época é caracterizado por fatores subjetivos e um certo amadorismo. Por exemplo, a participação do médico em congressos em que se apresentavam os resultados da utilização das tecnologias precipitava mecanismos de pressão para que o hospital adquirisse e implementasse as novas técnicas, independentemente do equacionamento das questões de aplicações e vocações estratégicas.

Em relação ao hospital estudado é possível a identificação de três fases distintas na abordagem do processo de incorporação. A primeira, que ocorre na consolidação organizacional, durante os anos 80-85. A segunda , com o reconhecimento do hospital como centro de excelência em alta complexidade, no período de 86 a 94. E a terceira, quando em função de mudança de sua estrutura de governança corporativa, no período subseqüente, o hospital introduz novos conceitos de avaliação e cria instância especificamente responsávelpelo processo.

Durante a primeira fase o hospital utilizava-se de mecanismos semelhantes aos de outros hospitais da cidade. Pela característica de possuir corpo clínico aberto (mantida até a atualidade), a presença de alguns médicos de grande reputação e propiciava condições de forte capacidade de interferência com as decisões de compras de novos equipamentos . Devido ao fato destes profissionais constituírem o núcleo mais importante de encaminhamentos para internações, a administração do hospital procurava criar condições de trabalho de forma a desenvolver a fidelização desta parte do corpo clínico à organização. As decisões que envolviam aquisição a introdução de novas técnicas passavam quase que obrigatoriamente pela ausculta das opiniões deste grupo inexistindo, em termos práticos, mecanismos de aferição acerca da pertinência e do impacto que destas incorporações.

Com a ascensão ao controle corporativo de um grupo que contava com a presença de um especialista com grande interesse em tecnologia médica, o hospital passa por uma mudança importante no que se refere às decisões. Estas passam a se concentrar no conhecimento e na experiência deste profissional e, nesta segunda fase, o hospital desenvolve um novo modelo de avaliação das tecnologias. Novos critérios de estudo do potencial das tecnologias no passam a integrar as discussões, entre os quais, as competências necessárias à operação, as necessidades de suporte técnico, o potencial de produção e as conseqüentes questões de custos e receitas e a identificação dos conhecimentos necessários aos médicos referenciadores acerca das indicações e aplicações. Nesta fase o hospital criou várias estruturas administrativas e de suporte que possibilitaram uma melhor utilização da planta tecnológica que desenvolvia. Por exemplo, criou-se um serviço de engenharia clínica, o primeiro do país, cujos propósitos foram o controle dos custos de manutenção e a redução dos tempos de parada dos equipamentos, estruturou-se junto ao centro de estudos um programa de educação médica com enfoque na utilização das novas tecnologias e incorporaram-se novas tecnologias com alta complexidade de sistemas de informações, como por exemplo, os novos equipamentos de ressonância magnética nuclear e o centro de radioterapia.

Com estas mudanças o hospital passa a ser identificado pelo mercado como o mais bem equipado da cidade e com a maior capacidade resolutiva entre hospitais semelhantes. Também, em decorrência desta avaliação dos usuários, amplia-se sua capacidade operacional e aumenta o contingente de médicos credenciados habilitados para tratamentos de alta complexidade com conseqüente mudança no mix de serviços. Esta fase se caracterizou pelo crescimento da planta física e pelo desenvolvimento de novas competências assistenciais, fenômenos eminentemente influenciados pela identificação do hospital pelo mercado como centro de excelência. 
Entretanto, as mudanças de cenário citadas anteriormente e a emergência de restrições ao crescimento organizacional desencadearam na liderança corporativa um processo de discussão acerca das estratégias a serem seguidas nos novos tempos. A organização estabelece novos objetivos estratégicos, passa por uma reestruturação administrativa com a criação de instâncias de controle e procede-se a profissionalização de su administração com transferência de responsabilidades antes adstritas à diretoria executiva para o grupo profissional que assume a gestão.

Nesta terceira fase é criada uma comissão de tecnologia médica composta por representantes da administração profissional, do corpo clínico, de profissionais ligados à engenharia hospitalar e de membros da diretoria executiva com o propósito de estruturar o processo decisório. Esta comissão organizou seu trabalho dentro de premissas que permitissem o encaminhamento das opções de incorporação de forma a otimizar a utilização do parque e o atendimento de processos assistenciais de maior valor agregado. Em conseqüência tornaram-se necessárias informações relacionadas a nosoepidemiologia hospitalar, às novas tendências de utilização de tecnologias, aos custos operacionais e de manutenção, às características tecnológicas dos principais hospitais concorrentes e ao modelo de remuneração utilizado pelas principais fontes pagadoras, clientes do hospital.

De posse destes dados, tornou-se possível à comissão, a introdução de critérios que permitissem à administração, a elaboração de projeções sobre fluxos de caixa futuros. Além disto, abriu-se a possibilidade de identificação de outros atributos necessários à decisão, como por exemplo, a identificação de tecnologias essenciais à atenção médica e uma classe de tecnologias relacionada aos aspectos de segurança do paciente, do trabalhador do hospital e do meio ambiente.

Com a implantação do trabalho da comissão, o conhecimento criado acerca do parque tecnológico e das necessidades de investimentos em sua atualização e manutenção permitiu a diretoria executiva o desenvolvimento metodologias de formulação de orçamentos, projeções de utilização, identificação de novos negócios e a adequações das estruturas operacionais e de suporte. Da mesma forma, questões relacionadas à qualidade dos serviços prestados, especialmente no que se refere à assertividade de sua utilização, e ao desenvolvimento de novos conhecimentos médicos se apresentaram como novas possibilidades ao corpo clínico e tem se constituído em elemento diferenciador do hospital em relação à sua concorrência.

\section{Fatores importantes para um modelo racional de incorporação}

Tendo em vista os elementos citados nos itens anteriores é possível o estabelecimento de uma grade de fatores necessários ao planejamento e à definição das melhores opções de tecnologias a serem incorporadas e conseqüentemente buscar informações que permitam a análise da utilização potencial. Em principio, podemos identificar os seguintes fatores e atributos:

1. Mercado - Identificação de população usuária e do seu potencial de consumo.

2. Identificação de capacidade interna de incorporação.

3. Potencial de concorrência disponível em outros agentes prestadores de serviços.

4. Características técnicas da incorporação. 
5. Capacidade de produção disponível, custos e receitas adicionadas e potencial de retorno que a incorporação propicia.

Com relação ao item 1 - Mercado - Identificação de população usuária e do seu potencial de consumo as informações necessárias se referem ao perfil social e epidemiológico da população alvo, com conseqüente estimativa de demanda potencial pela tecnologia em estudo. Além disto, quais as formas mais rotineiras de remuneração pelos procedimentos, os principais agentes compradores dos serviços e o contexto técnico em que tal tecnologia é demandada. Com estes dados é possível a inferência do potencial de ocupação do serviço bem como de quem detém o conhecimento técnico de solicitação e indicação de uso.

Com relação ao item 2 - Identificação da capacidade interna de incorporação, as informações se referem à inserção da tecnologia no contexto das vocações estratégicas da organização, à capacidade de operação disponível, ao potencial de substituição de outras tecnologias, à disponibilidade de pessoal habilitado e de recursos físicos para a implementação bem como das necessidades de recursos financeiros a serem disponibilizados.

Com relação ao item 3 - Potencial de concorrência disponível em outros agentes prestadores de serviços, as informações se referem à existência de tecnologia similar em outras organizações e à sua utilização bem como das políticas de preços praticados por estes concorrentes.

Com relação ao item 4 - Características técnicas da incorporação, as informações se referem à capacidade técnica dos fornecedores, de sua planta de manutenção, dos aspectos técnicos de suporte necessários pelos novos equipamentos e do potencial de atualização que a tecnologia propicia diante de inovações, especialmente em relação a sistemas de informações.

Com relação ao item 5 - Capacidade de produção disponível, custos e receitas adicionadas e potencial de retorno que a incorporação propicia, as informações se referem ao desenho e dimensionamento operacional com conseqüente cálculo dos valores a serem investidos, de seu custeio, de seu potencial de receitas e da identificação dos fatores de restrição de forma a permitir cálculos de desempenhos financeiros esperados.

De posse destas informações torna-se possível estabelecer classificação com base em critérios de segurança, essencialidade e rentabilidade que dará suporte ao processo decisório.

\section{Classificação dos investimentos em tecnologia médica}

Investimentos classe I - Cujo caráter principal é a criação de melhores condições de segurança ao paciente, ao trabalhador e ao ambiente físico, constituindo-se portanto em elemento fundamental ao processo assistencial. Constituem exemplos, as tecnologias de monitoração e controle de outras tecnologias médicas, de prevenção e combate a incêndios e de garantia de suprimentos de energia, água e de manutenção de equipamentos ligados a funções vitais.

Investimentos classe II - Caracterizados pela sua essencialidade, quer seja esta vinculada a um processo intermediário ou final da assistência. São exemplos deste grupo as tecnologias de diagnóstico por imagem, os sistemas de suporte ventilatório, os equipamentos utilizados em 
procedimentos terapêuticos e intervencionistas e aqueles que direta ou indiretamente sejam responsáveis pela produção assistencial. Neste grupo, é possível ainda uma subclassificação em função do caráter de rentabilidade que a tecnologia propicia.

Investimentos Classe III- Caracterizados pela sua não essencialidade ao processo de assistência, sendo necessário um pareamento de suas incorporações com as vocações assistenciais da organização.

Investimentos Classe IV - Caracterizados pela sua aplicação em pesquisa médica e desenvolvimento de novos serviços e produtos, e que apresentam um grau maior de dificuldade de análise posto que se referem a procedimentos ainda não bem estabelecidos no ambiente assistencial.

\section{CONCLUSÃO}

As organizações de saúde têm se defrontado com crescentes desafios e dificuldades. Em um ambiente de restrições, em que as pressões dos vários agentes envolvidos com o sistema se estabelecem em regimes permanentes, a questão da incorporação de novas tecnologias demanda novos posicionamentos e a administração de um volume muito maior de informações que subsidiem o processo decisório. Entre estas, é possível selecionar dados relativos à evolução epidemiológica e demográfica da população, aos padrões de remuneração praticados pelo mercado comprador de serviços e à capacidade de absorção e aprendizagem disponíveis nos grupos profissionais do hospital no sentido de compor um modelo de análise que estabeleça a utilidade e aplicação das novas tecnologias.

Da mesma forma, o monitoramento das tendências de inserção de novas tecnologias, especialmente as que demandem mudanças de comportamento médico e que necessitem de sua participação na implementação de novos processos assistenciais, deve se constituir em item de preocupação permanente dos planejadores e gestores de organizações de assistência médico-hospitalar.

Neste sentido, a utilização de um modelo que conjugue dados relacionados a vários setores do conhecimento pode permitir a redução da incerteza quanto à adequação dos programas de investimentos em tecnologia, preservando a organização e seus papéis no âmbito da comunidade em que se insere. Trabalhar com conceitos que privilegiem os critérios de essencialidade, de preservação da segurança ambiental e do paciente, de rentabilidade e de participação do médico referenciador pode vir a se constituir em mecanismo válido de justificação do planejamento em incorporação de tecnologia. 


\section{BIBLIOGRAFIA}

ANDERSON, H. Hospitals Seek New Ways to Integrate Health Care, Hospitals and Health Networks, $1992, n^{\circ} 7$, p. $26-36$.

ANDREWS et al. Organizational Transformation in Health Care. San Francisco: Jossey-Bass, 1994.

AUGIER, E. et al. L'hôpital et les nouvelles technologies. Hôpital dïlle de France. Paris, 2000, Vol.25, S 2136 .

BARBOSA, A.P. Qualidade em Serviços de Saúde. Dissertação apresentada a EAESP-FGV, São Paulo, 1995.

BAIG, E. The information Society. bonus, Business Week. may 18,1994, p.122-133.

BANGA, B. La Révolution Technologique à l'Hôpital. Décision Santé, Paris, 1997.

BRONZINO, J.D. The Biomedical Engineering Handbook. CRC Press, 1995.

CHAVES, M.M. Saúde, Uma Estratégia de Mudança. Ed.Guanabara, Rio de Janeiro, 1982.

CONSTITUIÇÃO DA REPÚBLICA FEDERATIVA DO BRASIL, Artigos 196, 198, 199 e 200. Brasília, 1988.

DE WOLF, L e GILOTH,B. Strategies for Outcomes Measurements: How Seven Systems Do It. Healthcare Forum, 1998, 41(4), p.32-34

LANDMANN, J. Evitando a Saúde e Prevenindo a Doença. Achiamé, Rio de Janeiro, 1982.

MYCEK, S. Leadership for a Healthy $21^{\text {st }}$ Century. Healthcare Forum, 1998, 41(4), p.26-31

ORGANIZACIÓN PANAMERICANA DE LA SALUD. El Desarrollo de la Evaluación de las Tecnologias en Salud en America Latina y Caribe. OPS, Washington, DC, 1998.

PICCHIAI, D. Mudanças em Instituições Hospitalares: Análise do Processo e Estudo da Ação do Administrador Hospitalar. Relatório de Pesquisa apresentado ao NPP - Núcleo de Pesquisas e Publicações - EAESP-FGV. São Paulo, 1998.

PORTER-O"GRADY, T., WILSON, C.K. The Leadership Revolution in Health Care - Altering Systems, Changing Behaviors. Aspen Publication, Gaitesburg, MD, 1995.

SOPER, M.R., et al. Balancing the Triad: Cost Containment, Quality of Service, and Quality of Care in Managed Care Systems. National Center for Managed Health Care Administration, University of Missouri, 1995.

TONGES, M.C. Works Designs in Sociotechnical Systems for Patient Care Delivery. Nursing Management, 1992, v.23, no 9 , p.50-51. 